\title{
Generation of attosecond soft x-ray pulses in a longitudinal space charge amplifier
}

\author{
M. Dohlus, E. A. Schneidmiller, and M. V. Yurkov \\ Deutsches Elektronen-Synchrotron (DESY), Notkestrasse 85, D-22607 Hamburg, Germany
}

(Received 30 March 2011; published 14 September 2011)

\begin{abstract}
A longitudinal space charge amplifier (LSCA), operating in soft x-ray regime, was recently proposed. Such an amplifier consists of a few amplification cascades (focusing channel and chicane) and a short radiator undulator in the end. The broadband nature of LSCA supports generation of few-cycle pulses as well as wavelength compression. In this paper we consider an application of these properties of LSCA for generation of attosecond x-ray pulses. It is shown that a compact and cheap addition to the soft x-ray freeelectron laser facility FLASH would allow one to generate 60 attosecond (FWHM) long x-ray pulses with the peak power at the $100 \mathrm{MW}$ level and a contrast above $98 \%$.
\end{abstract}

DOI: 10.1103/PhysRevSTAB.14.090702

PACS numbers: 41.60.Cr

\section{INTRODUCTION}

Longitudinal space charge (LSC) driven microbunching instability $[1,2]$ in electron linacs with bunch compressors (used as drivers of short wavelength FELs) was a subject of intense theoretical and experimental studies during the past years [3-10]. Such instability develops in infrared and visible wavelength ranges and can hamper electron beam diagnostics and free-electron laser (FEL) operation.

It was proposed in [11] to use this effect for generation of vacuum ultraviolet (VUV) and $\mathrm{x}$-ray radiation. A concept of a longitudinal space charge amplifier (LSCA) was introduced, scaling relations for an optimized LSCA were obtained, and its possible applications were analyzed. It was pointed out, in particular, that a broadband nature of LSCA supports generation of attosecond pulses.

There are many different schemes for generation of attosecond $\mathrm{x}$-ray pulses from free-electron lasers (FELs) [12-19]. Most of the proposed schemes make use of a short intense laser pulse to manipulate electron energy in a small slice of an electron bunch and to select only this short slice with the help of a FEL mechanism.

In Ref. [11], a scheme was suggested that uses the longitudinal space charge amplifier in combination with laser manipulation of the electron beam to produce attosecond soft $\mathrm{x}$-ray pulses. In this paper we describe the operation of the LSCA-based attosecond scheme in detail, exemplify it with the parameters of the soft x-ray free-electron laser facility FLASH [20,21], perform thorough numerical simulations, and discuss a possible implementation at FLASH making use of the existing infrastructure.

Published by the American Physical Society under the terms of the Creative Commons Attribution 3.0 License. Further distribution of this work must maintain attribution to the author(s) and the published article's title, journal citation, and DOI.

\section{OPERATION OF THE LONGITUDINAL SPACE CHARGE AMPLIFIER}

The scheme (see Fig. 1) of the longitudinal space charge amplifier (LSCA) [11] is simple both conceptually and technically. An amplification cascade consists of a focusing channel and a dispersive element (usually a chicane) with an optimized momentum compaction $R_{56}$. In a channel the energy modulations are accumulated, which are proportional to density modulations and space charge impedance of the drift space. In the chicane these energy modulations are converted into induced density modulations that are much larger than initial ones [1], the ratio defines a gain per cascade. In this paper we will consider the case when the amplification starts up from the shot noise in the electron beam. A number of cascades are defined by the condition that the total gain, given by the product of partial gains in each cascade, is sufficient for saturation (density modulation on the order of unity) after the start-up from shot noise. The amplified density modulation has a large relative bandwidth, typically in the range $50 \%-100 \%$. Behind the last cascade a radiator undulator is installed, which produces a powerful radiation with a relatively narrow line (inverse number of periods) within the central cone. This radiation is transversely coherent, and the longitudinal coherence length is given by the product of the number of undulator periods by the radiation wavelength. When LSCA saturates in the last cascade, a typical enhancement of the radiation power over that of spontaneous emission is given by a number of electrons per wavelength.

\section{DESCRIPTION OF THE LSCA-BASED ATTOSECOND SCHEME}

The first few cascades of LSCA (as in Fig. 1) operate as described above. A broadband density modulation is amplified around the optimal wavelength

$$
\lambda_{0} \simeq 2 \pi \sigma_{\perp} / \gamma,
$$




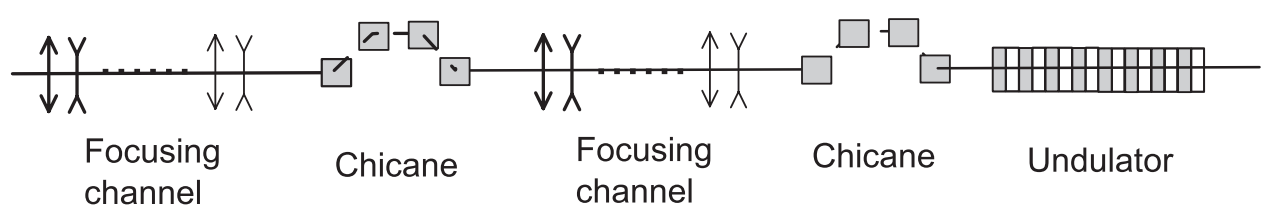

FIG. 1. Conceptual scheme of an LSC amplifier.

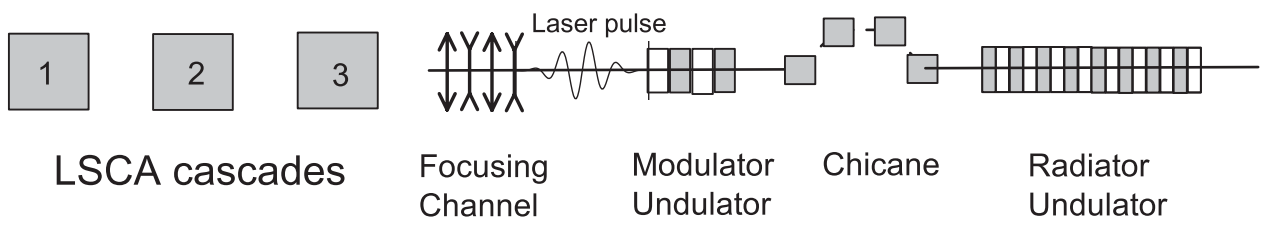

FIG. 2. LSCA-based attosecond scheme. Numbered boxes in the left part of the figure denote standard cascades of LSCA (focusing channel plus chicane).

where $\sigma_{\perp}$ is a transverse size of the beam, and $\gamma$ is relativistic factor. Such a choice of the wavelength is optimal because the LSC impedance reaches its maximum, and, at the same time, transverse correlations of the LSC field are on the order of the beam size [4], which guarantees a good transverse coherence of the radiation in the end. Length of a drift space [22], beta function, and momentum compaction $R_{56}$ of a chicane are optimized for the chosen wavelength.

The last cascade is modified as shown in Fig. 2. A short two-period modulator undulator is installed in front of the last chicane. In this undulator the electron beam is modulated in energy (modulation wavelength is much longer than $\lambda_{0}$ ) by a few-cycle powerful laser pulse [23] in the same way as it is suggested for FEL-based attosecond schemes [13,14,16-19] (see Fig. 3 as an example). A short slice in the electron bunch (between 0 and $0.3 \mu \mathrm{m}$ in Fig. 3) gets the strongest energy chirp, and is being strongly compressed (by a factor $C \gg 1$ ) in the following

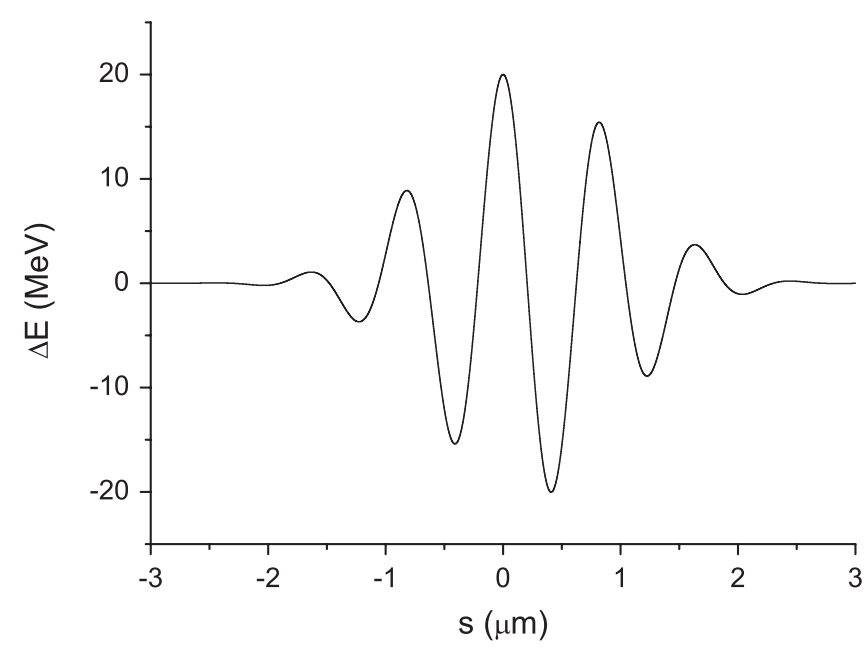

FIG. 3. Energy modulation, induced on the beam by a short laser pulse in a two-period undulator. Energy deviation is plotted as a function of the longitudinal position in an electron bunch. chicane. The $R_{56}$ of this chicane and the chirp are adjusted such that a required wavelength compression within that slice is achieved, and, at the same time the amplification of the microbunching within the slice through the last chicane is optimal (parameters of the whole amplification chain are adjusted such that saturation is reached in the last chicane). Other parts of the electron bunch are either uncompressed or have much weaker compression than the slice with the strongest energy chirp.

The chicane is followed by a short radiator undulator of which resonance wavelength, $\lambda_{0} / C$, corresponds to the (broadband) wavelength spectrum within the strongly compressed slice. Then only this short slice of the electron bunch produces undulator radiation within the central cone (which is selected by a pinhole downstream), while modulation wavelengths in the rest of the electron bunch are much longer than the resonance wavelength of the undulator. In other words, the rest of the electron bunch produces only spontaneous emission within the central cone, and this emission would define the contrast of generated attosecond pulses.

Finally, let us note that the broadband nature of LSCA makes wavelength compression especially attractive [11]. Indeed, the compression factor is given by the formula

$$
C=\left(1-h R_{56}\right)^{-1}
$$

where $h$ is the linear energy chirp (the derivative of relative energy deviation). For a large $C$ a variation of the compression factor reads

$$
\frac{\Delta C}{C} \simeq C \frac{\Delta h}{h}
$$

After the compression the bands of density modulations and of the radiator must overlap. This leads to the following requirement on the compression stability:

$$
\frac{\Delta C}{C}<\frac{\Delta k_{\max }}{k}
$$


where $\Delta k_{\max }=\max \left(\Delta k_{\mathrm{den}}, \Delta k_{\mathrm{rad}}\right)$, and $\Delta k_{\mathrm{den}}$ and $\Delta k_{\mathrm{rad}}$ are bandwidths of the density modulation and of the radiator, respectively. Thus, the stability of the chirp must satisfy the requirement

$$
\frac{\Delta h}{h}<\frac{1}{C} \frac{\Delta k_{\max }}{k} .
$$

For coherent FEL-type modulations and an undulator as a radiator $\Delta k_{\max } / k \ll 1$ which might set very tight tolerance for the chirp stability and limit practically achievable compression factors. For an LSCA, however, $\Delta k_{\max } / k=$ $\Delta k_{\mathrm{den}} / k \simeq 1$, so that for a given chirp stability one can go for much stronger compression. Alternatively, for a given compression factor, one can significantly loosen the tolerances. Note also that nonlinearities of the longitudinal phase space do not play a significant role in the case of LSCA.

\section{PARAMETER SET FOR AN ATTOSECOND LSCA}

We exemplify an operation of the attosecond scheme with the parameters of the soft x-ray FEL facility FLASH $[20,21]$. In Sec. VI we will discuss a possible technical implementation of this scheme making use of the existing infrastructure at FLASH.

Concerning the choice of parameters of the electron beam, we rely on the results of beam dynamics simulations $[24,25]$. For the case of the bunch charge $100 \mathrm{pC}$, we reduce compression by a factor of 2 (with respect to the scenario considered in [25]), thus ending up with the slice parameters presented in Table I.

Number of LSCA cascades, their parameters, and the operating wavelength range are chosen with the help of the guidelines of Ref. [11]. As a focusing structure we choose FODO lattice ${ }^{2}$ with the period $1.4 \mathrm{~m}$ and the average beta function also $1.4 \mathrm{~m}$ (even smaller beta function would be preferable, but the technical feasibility and operational issues have to be taken into account). For electron beam parameters from Table I, we calculate from (1) that the optimal wavelength for amplification in LSCA is around $40 \mathrm{~nm}$. The length of a drift in each cascade is chosen to be $2.8 \mathrm{~m}$, or two FODO periods. This length should provide a sufficient gain per cascade, it is smaller than a reduced wavelength of plasma oscillations, and the longitudinal velocity spread due to emittance does not play any role [11]. The optimal $R_{56}$ of the chicane for a given energy spread and wavelength range is about $50 \mu \mathrm{m}$. The chicane

TABLE I. Electron beam parameters.

\begin{tabular}{lc}
\hline \hline Energy & $1.2 \mathrm{GeV}$ \\
Charge & $100 \mathrm{pC}$ \\
Peak current & $1 \mathrm{kA}$ \\
Slice energy spread & $150 \mathrm{keV}$ \\
Slice emittance & $0.4 \mathrm{~mm} \mathrm{mrad}$ \\
\hline \hline
\end{tabular}

in each cascade should fit in a space between two quadrupoles. Thus, the total length of a cascade is about $3.5 \mathrm{~m}$ (or 2.5 FODO periods).

According to our estimates, we need three regular cascades with the parameters, described above, and a last special cascade (see Fig. 2), so that a total length of the system is about $14 \mathrm{~m}$. In the last cascade we have a drift of the same length $(2.8 \mathrm{~m})$, followed by a two-period undulator and a chicane with reduced $R_{56}$. The undulator has a period length of $10 \mathrm{~cm}$ and a peak field of $1.4 \mathrm{~T}$, so that a resonance of $1.2 \mathrm{GeV}$ beam with the laser beam (wavelength is $800 \mathrm{~nm}$ ) is provided. In the last cascade a final amplification and, at the same time, a wavelength compression (down to $4-5 \mathrm{~nm}$ ) in a short slice take place. To estimate the required energy chirp and the $R_{56}$ of the last chicane, we have to take the following considerations into account. The amplitude of density modulation at the compressed wavelength should not be strongly suppressed by the uncorrelated energy spread during compression. For a Gaussian energy distribution an amplitude gain per cascade is given by [1]

$$
G=C k_{0}\left|R_{56}\right| \frac{I}{\gamma I_{\mathrm{A}}} \frac{4 \pi\left|Z\left(k_{0}\right)\right| L_{d}}{Z_{0}} \exp \left(-\frac{1}{2} C^{2} k_{0}^{2} R_{56}^{2} \frac{\sigma_{\gamma}^{2}}{\gamma^{2}}\right) .
$$

Here $k_{0}=2 \pi / \lambda_{0}$ is the modulation wave number before compression, $Z$ is the impedance of a drift space (per unit length), $Z_{0}$ is the free-space impedance, $L_{d}$ is the length of the drift space, $I$ is the beam current, $I_{\mathrm{A}}$ is the Alfven current, $\gamma$ is relativistic factor, and $\sigma_{\gamma}$ is rms uncorrelated energy spread (in units of rest energy). The beam current and energy spread are taken before compression. The optimal $R_{56}$ from (6) is given by $R_{56} \simeq$ $\gamma\left(\sigma_{\gamma} k_{f}\right)^{-1}$, where $k_{f}=C k_{0}$ is the modulation wave number after compression. Then the required energy chirp can be defined from (2):

$$
h=\frac{C-1}{C R_{56}} \simeq \frac{C-1}{C} \frac{\sigma_{\gamma}}{\gamma} k_{f} .
$$

If the electron beam is modulated by a laser with the wavelength $\lambda_{L}=2 \pi / k_{L}$, and the modulation amplitude is $\delta \gamma$, then the chirp at zero crossing is $h \simeq k_{L} \delta \gamma / \gamma$. Thus, with the help of (7) we get an estimate of a required amplitude of energy modulation:

$$
\delta \gamma \simeq \frac{C-1}{C} \frac{\lambda_{L}}{\lambda_{f}} \sigma_{\gamma}
$$

If the compression factor is about $8-10, \lambda_{L}=800 \mathrm{~nm}$, $\lambda_{f} \simeq 5 \mathrm{~nm}$, then the required energy modulation amplitude is about $20 \mathrm{MeV}$, and the corresponding $R_{56}$ is $6-7 \mu \mathrm{m}$. We should note that formula (6) is valid for a linear regime of amplification, while we deal with a saturation in the last

\footnotetext{
${ }^{2}$ FODO lattice indicates periodic focusing structure with the period: focusing quadrupole, drift, defocusing quadrupole, drift.
} 
cascade. Moreover, energy modulations due to a short laser pulse are not sinusoidal. Therefore, the above obtained estimate can only be used as a first guess. For our simulations we choose the modulation amplitude of $20 \mathrm{MeV}$, and the $R_{56}$ was optimized in the simulations at $7.1 \mu \mathrm{m}$. In order to get the desired energy modulations, we use a laser pulse with the duration of $5 \mathrm{fs}$ (FWHM) and pulse energy $3 \mathrm{~mJ}$. The laser beam is focused into the modulator undulator, the spot size in the waist is $w_{0}=300 \mu \mathrm{m}$.

Finally, we choose parameters of the radiator undulator that should be tuned to the desired wavelength of $5 \mathrm{~nm}$ for the given beam energy of $1.2 \mathrm{GeV}$. We take a planar undulator with the following parameters: undulator period is $2.5 \mathrm{~cm}$, peak field is $0.67 \mathrm{~T}$, number of periods is 5 . We assume that the undulator has a tunable gap which would allow us to change wavelength by changing the gap and/or the beam energy.

\section{NUMERICAL SIMULATIONS OF THE ATTOSECOND LSCA}

The numerical simulations of the LSCA operation were done as follows. Self-interaction of electrons in the beam by space charge fields in the focusing channels was simulated with a $3 \mathrm{D}$ version of the space charge tracking code ASTRA [26,27]. All important aspects of the problem like betatron motion of particles in the FODO channel, threedimensional calculation of the space charge field, and startup from shot noise were included in the simulations. To properly treat the start-up from noise, we took only a short part of the bunch with the length about $2 \mu \mathrm{m}$, and used a real number of particles, $4 \times 10^{7}$, distributing them randomly in 6D phase space. After the energy change of each particle in a drift was calculated, we simply applied $R_{56}$ to add the effect of a chicane (later we checked that CSR effects do not play any significant role, see below). In this

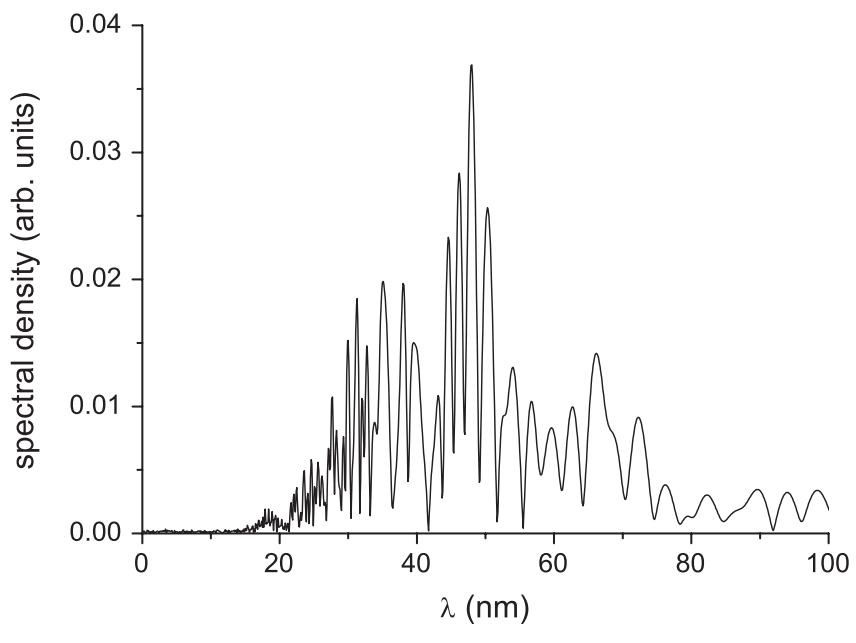

FIG. 5. Spectrum of the modulated electron current (modulus of the bunch form factor) after the third amplification cascade. The calculations were performed with ASTRA.

way the evolution of the particle distribution through a single cascade was simulated. Then the procedure was repeated for the next cascade, etc. The results of simulations of the first three cascades of LSCA are shown in Figs. 4 and 5. In Fig. 4 we show the amplified noisy modulations of beam current, and in Fig. 5 the modulus of the bunch form factor (Fourier transform of the trace in Fig. 4, left) is presented. Note that a linear gain (in amplitude) per cascade is about 5 at $40-50 \mathrm{~nm}$ wavelength. In the third cascade the amplification becomes slightly nonlinear, so that the gain is reduced by about $10 \%$. Typical feature size in spectrum (interval of spectral coherence) can be estimated as $\Delta \omega \simeq 2 \pi c / l_{b}$ [28], where $l_{b} \simeq 2 \mu \mathrm{m}$ is the length of a part of the bunch in our simulations. Converting this dependence to a wavelength interval, we get $\Delta \lambda \simeq$ $\lambda^{2} / l_{b}$, i.e., one should expect a quadratic dependence of the coherence interval on the wavelength. Also note that

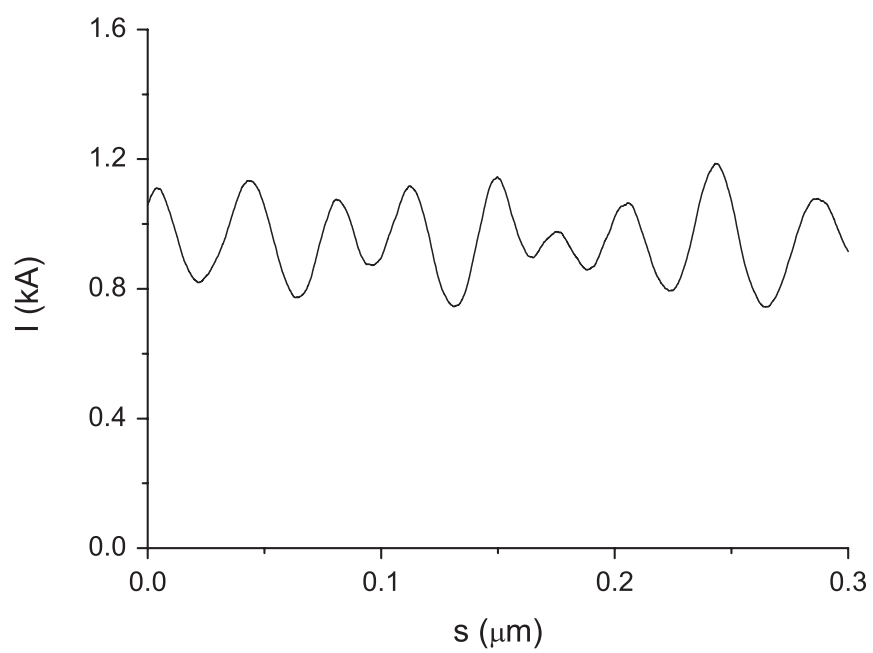

FIG. 4. Modulated electron bunch (left) and its enlarged fraction (right) after the third amplification cascade. The calculations were performed with ASTRA. 

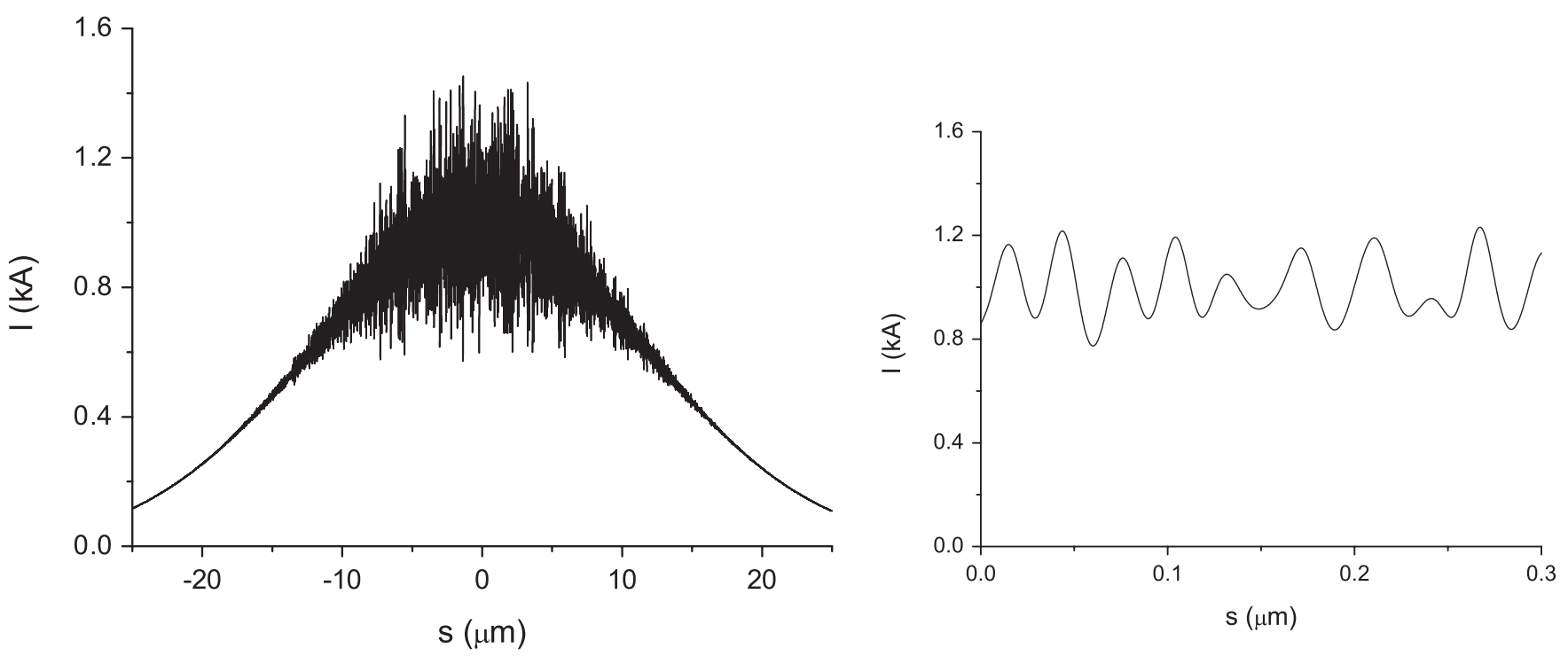

FIG. 6. Modulated electron bunch (left) and its enlarged fraction (right) after the third amplification cascade. The calculations were performed with LOSCA.

in case of simulations with ASTRA we used only a short part of a $100 \mathrm{pC}$ bunch, so that the fine structure in spectrum does not correspond to a real situation with much longer bunch.

Alternatively, we have developed a simple and fast 1D code LOSCA (longitudinal space charge amplifier). Initially, particles are distributed randomly in the phase space time energy. The beam is then cut into short slices (much shorter than $\lambda_{0} / 2 \pi$ ), and the LSC interaction between the slices in a drift space is simulated with the help of the LSC wake function, averaged over beam cross section [29]. The drift is divided into pieces, and at the end of each piece the particles change their positions depending on their energies. The change of particles' distribution through a chicane is simply performed by applying $R_{56}$. Note that in our particular case the change of particles' positions happens only in chicanes. In the drifts it is negligibly small because of a very small spread of the longitudinal velocities due to both energy spread and emittance, and also because the length of a drift is much smaller that the reduced wavelength of plasma oscillations [11]. This means that for our parameter set it is sufficient to calculate only energy change of particles due to LSC interaction without dividing the drift into pieces. In fact, due to a simple model, used in LOSCA, we were able to relatively quickly calculate the evolution of particles' distribution through LSCA cascades with real number of particles in a bunch (with the charge of $100 \mathrm{pC}$ ), namely, with $6 \times 10^{8}$ particles. To have a representative set of data, we performed several simulation runs with different initial shot noise realizations. The results of
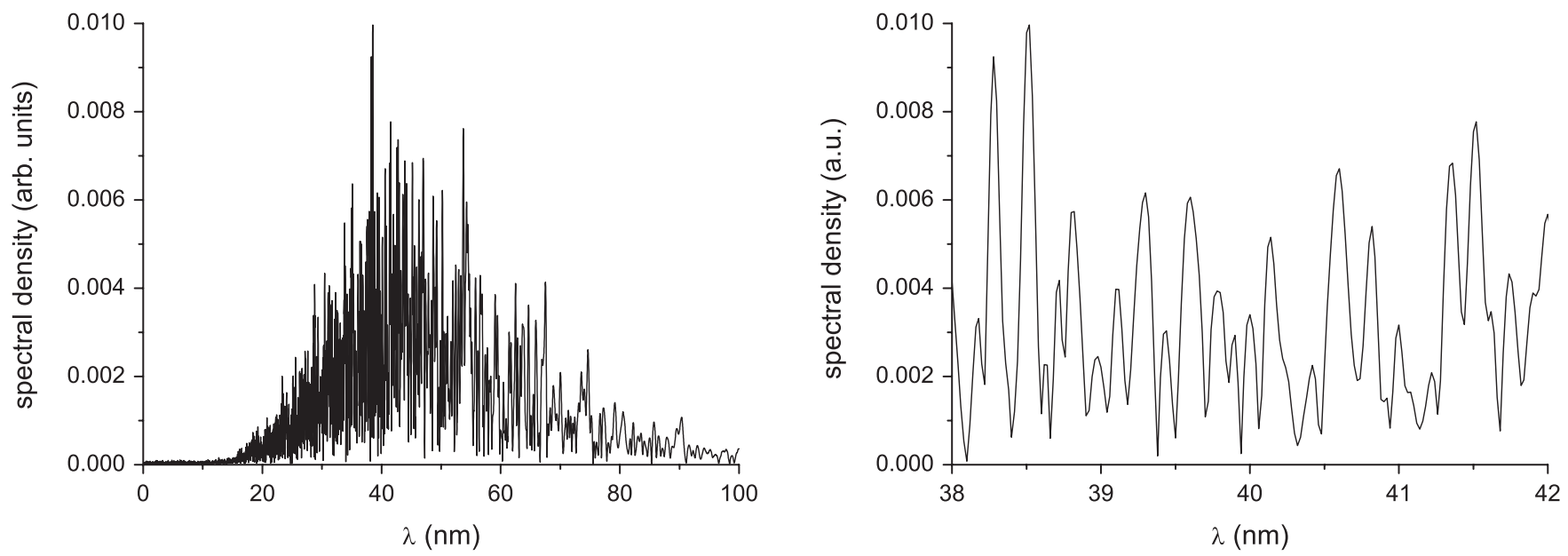

FIG. 7. Spectrum of the modulated electron current (modulus of the bunch form factor) and its enlarged fraction after the third amplification cascade. The calculations were performed with LOSCA. 

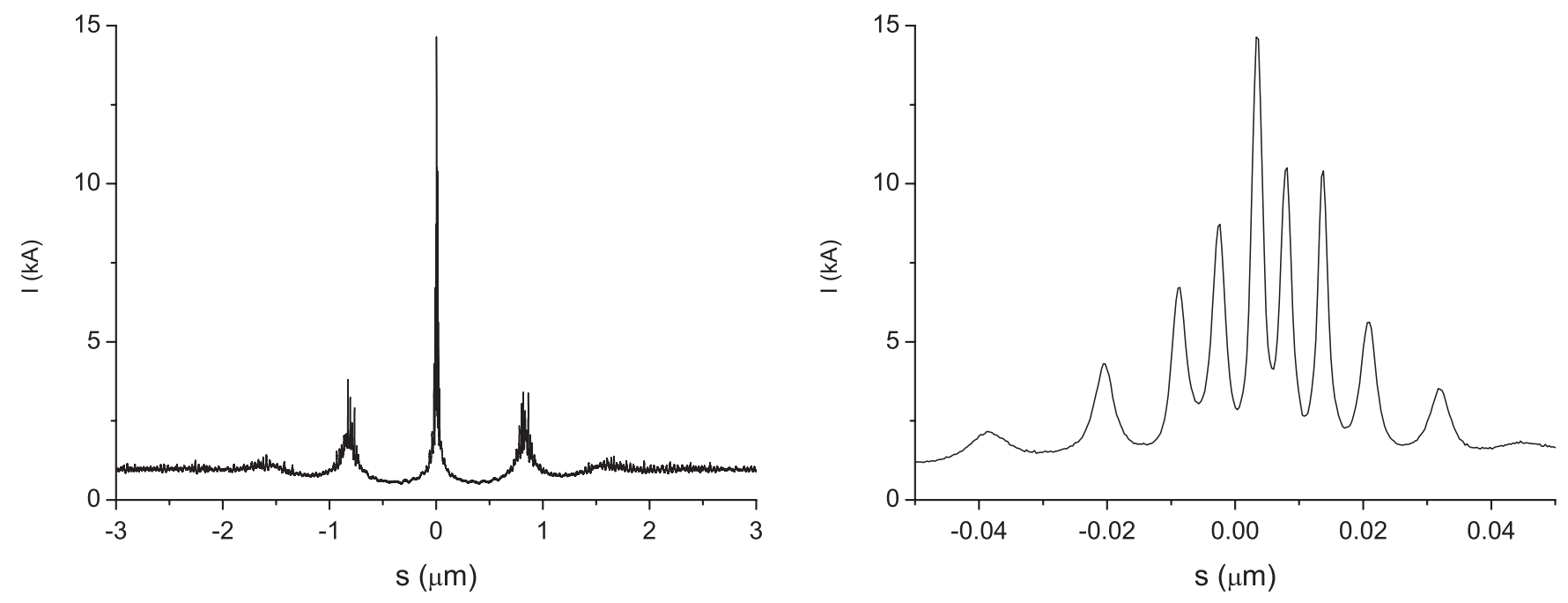

FIG. 8. Central part of the electron bunch (left) and a zoomed high-current part (right) after compression in the fourth chicane. The calculations were performed with LOSCA.

simulations of the first three cascades of LSCA for one of realizations are presented in Figs. 6 and 7.

It is interesting to observe (see right plots in Figs. 4 and 6) that LOSCA gives the same amplification of shot noise modulations as ASTRA, despite the fact that essentially different models are used in these two codes. We propose the following explanation. For a considered parameter range a typical transverse size of the LSC field is comparable to the electron bunch transverse size. Particles in ASTRA execute betatron oscillations and sample different LSC fields, while in LOSCA there is no transverse motion but the applied LSC wake is averaged over electron beam cross section. Our conclusion is that both approaches lead to very similar results for the LSCA gain, so that a

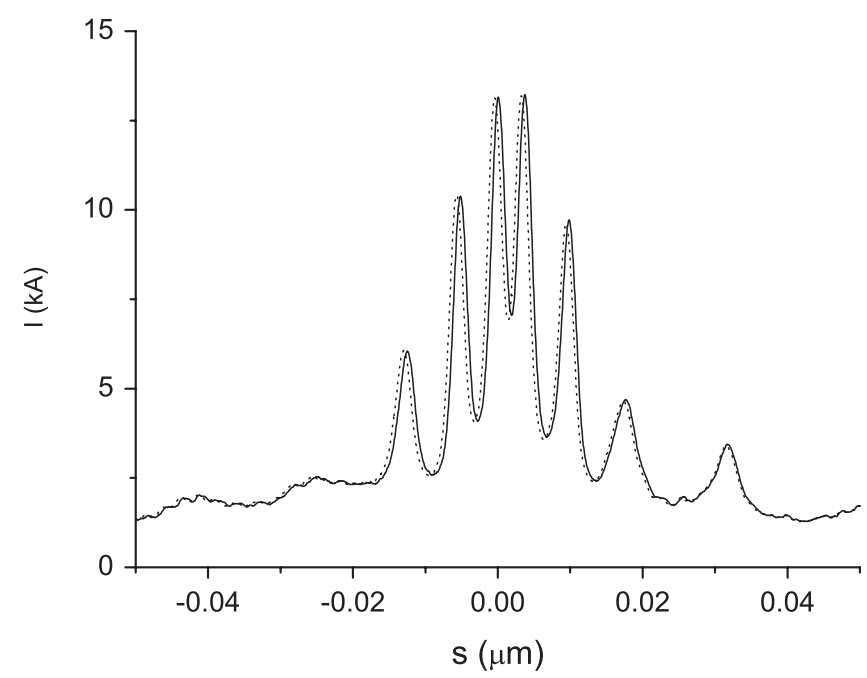

FIG. 9. Results of simulations with CSRTRACK and ASTRA. Zoomed high-current part of the bunch after the fourth chicane for the cases when CSR is off (solid line) and on (dots). The input distribution was simulated with ASTRA (but the number of particles was reduced in CSRTRACK simulations). simplified approach is justified for a typical parameter range of LSCA [11]: amplified wavelength is in the range described by Eq. (1), and beta function is smaller than a length of a drift space in an amplification cascade.

In the fourth amplification cascade the beam first moves in the $2.8 \mathrm{~m}$ long drift, accumulating energy modulation due to LSC field, and then it is modulated in energy by a short laser pulse. Modulation of the beam by a laser pulse in two-period undulator was simulated in the same way as it was done in $[12,13,17]$, namely, by a direct integration of equations of motion in the combined field of laser and undulator. The resulting energy modulation is presented in Fig. 3, it was imposed on the phase space distribution of the particles coming from the last drift. Then the effect of the last chicane was simulated by applying $R_{56}=7.1 \mu \mathrm{m}$. A typical current distribution for the beam, simulated with LOSCA, is presented in Fig. 8. Of course, amplitudes and positions of peaks (right plot in Fig. 8) are changing shot to shot. A similar distribution is obtained from the simulation with ASTRA, see Fig. 9.

The next problem is a possible influence of coherent synchrotron radiation (CSR) field in the chicane on extremely short high-current spikes. We have performed simulations with the code CSRTRACK [30,31]. We took the particle distribution at the exit of the fourth drift (simulated with ASTRA), imposed energy modulation by a laser, reduced number of particles (still having representative ensemble), and transferred this distribution into CSRTRACK. Finally, we did tracking through the chicane, taking into account CSR wake with the help of the projected model [32,33]. The results can be seen in Fig. 9. As expected [11], no noticeable effects on longitudinal and transverse dynamics were observed (except for a little time shift). The reason for this (as discussed in detail in [11]) is a practically complete smearing of microstructures inside the chicane due to the coupling of transverse and longitudinal dynamics 


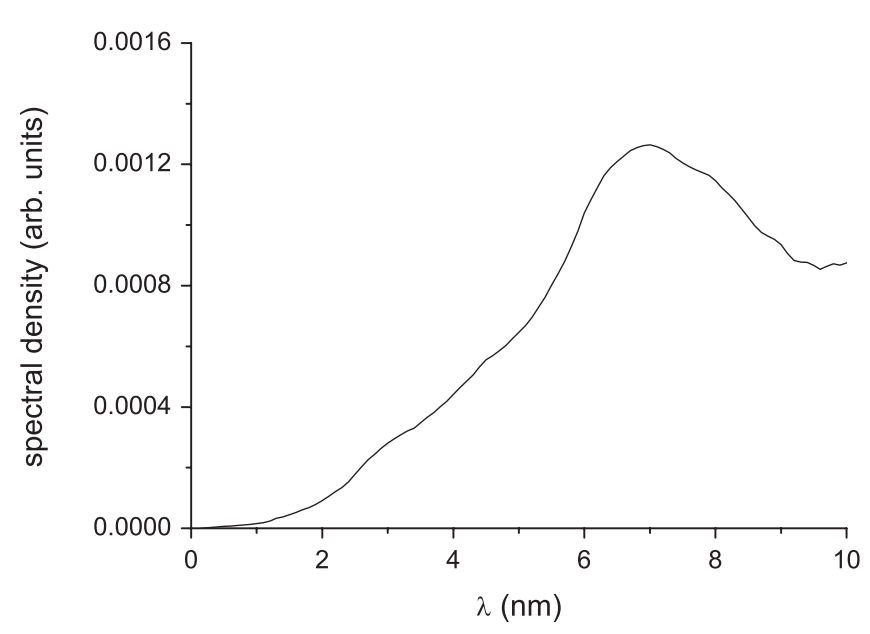

FIG. 10. Ensemble-averaged spectrum of the electron current (modulus of the bunch form factor) after the last chicane. The calculations were performed with LOSCA.

described by $R_{51}$ and $R_{52}$ elements of transfer matrix [34-36].

Ensemble-averaged spectrum of the beam current [37] after the last chicane is presented in Fig. 10. Within the spectral window, shown in this figure, the spectral components are dominantly coming from the fine structure within the main peak in Fig. 8 (right). The fine structures within two side peaks (which are significantly weaker compressed), contribute to the spectral density for wavelengths longer than $10 \mathrm{~nm}$. If a resonance wavelength of the radiator undulator is within the range of $2-8 \mathrm{~nm}$, one would get quite powerful radiation generated by fine structure within the main peak, i.e., very short pulses-depending also on the number of undulator periods. Some small contribution from side peaks is, in principle, possible due to a nonlinear harmonic generation mechanism during LSC amplification and compression.

To illustrate the main properties of the radiation, we assume that the undulator is tuned to $5 \mathrm{~nm}$, and the number of periods is 5 (undulator parameters are presented in the previous section). The radiation process was simulated with the code FAST [38]. For this purpose, the particles' distribution, simulated with the help of ASTRA or LOSCA, was transferred into FAST input distribution. We should note that FAST, as well as other FEL codes, uses resonance approximation, i.e., it deals essentially with narrow-band signals. The question arises wether or not one can properly simulate a process if the input signal (density modulation) has a broad band. We can answer this question as follows (see [28] for more details): within the central cone of the undulator radiation the code produces correct results even if the incoming density modulation has a wide band (in particular, if there is only shot noise having white spectrum). The accuracy of simulations of radiation properties within the central cone is on the order of inverse number of undulator periods, i.e., about $20 \%$ in our case. Main simulation results are presented in Fig. 11. Several typical realizations of attosecond pulses are shown, which were obtained from incoming particles' distributions, simulated with ASTRA (black line) and LOSCA. A typical duration of these pulses is 50-70 attoseconds (FWHM), the peak power is at $100 \mathrm{MW}$ level, and the bandwidth is about $20 \%$. Ensemble-averaged pulse energy is $5 \mathrm{~nJ}$ with the pulse-to-pulse rms fluctuations about $35 \%$. One can sometimes observe side peaks (corresponding to those in density modulation in Fig. 8, left plot), but they are typically below $1 \%$ of the power in the main peak. The rest of the bunch radiates only spontaneously (amplified density modulations in the range $20-80 \mathrm{~nm}$ could have produced radiation
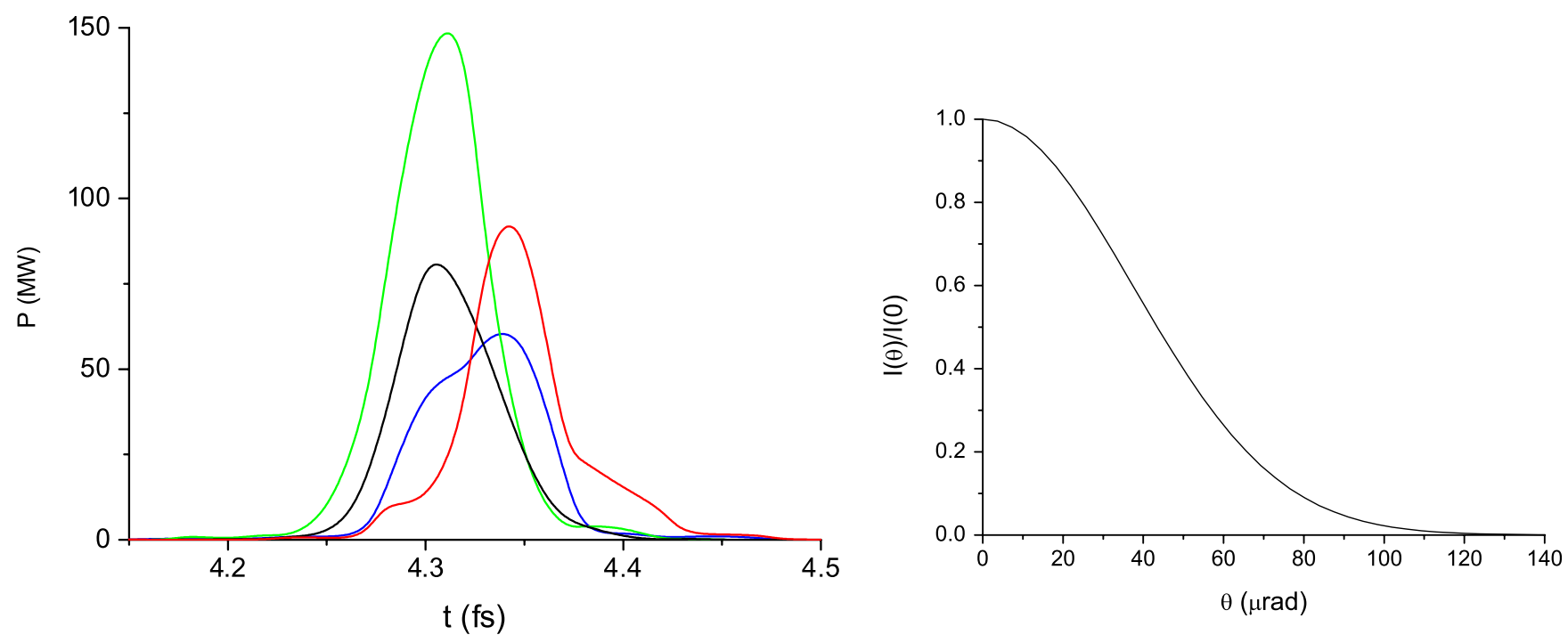

FIG. 11. The results of simulations of the radiation process in the last undulator with the code FAST when the undulator is tuned to $5 \mathrm{~nm}$. Left: different realizations of attosecond pulses. Amplification in LSCA was simulated with ASTRA (black) and LOSCA (red, blue, and green). Right: intensity distribution in far zone. 
into large angles, well beyond the central cone, but this radiation is totally suppressed due to a finite transverse size of the beam). As one can see from Fig. 11 (right plot), the half angle of coherent radiation is about $50 \mu \mathrm{rad}$. Selection (with the help of a pinhole) of a cone with half- angle 100-120 $\mu \mathrm{rad}$ would mean that one does not lose any power of attosecond x-ray pulses, while spontaneous radiation background is reduced to the level of $50 \mathrm{pJ}$ for $100 \mathrm{pC}$ bunch. Thus, we can state that a contrast (defined as a ratio of radiation energy of attosecond pulses to the total pulse energy) is high, above $98 \%$.

\section{IMPLEMENTATION OF THE ATTOSECOND SCHEME AT FLASH}

FLASH (Free-electron Laser in Hamburg) is the soft $\mathrm{x}$-ray FEL user facility $[20,21]$ operating in the wavelength range from 4 to $50 \mathrm{~nm}$. Short VUV and soft $\mathrm{x}$-ray pulses (down to $10 \mathrm{fs}$ [20]) are produced in a $27 \mathrm{~m}$ long undulator in self-amplified spontaneous emission (SASE) mode [39]. Recently, radiation at water window wavelengths down to $4 \mathrm{~nm}$ was generated. FLASH layout is shown in Fig. 12. The beam is produced by the laser-driven rf gun, compressed in two bunch compressors, and accelerated in superconducting rf modules up to $1.2 \mathrm{GeV}$. Then the beam is guided through the dogleg (where it is collimated), moves about $50 \mathrm{~m}$ towards SASE undulator, radiates there, and goes to the beam dump (alternatively, it can go to the dump via bypass).

Presently, there are two temporal experimental setups in the straight section between the dogleg and the undulator. Optical replica synthesizer (ORS) [41,42] is placed (not shown in Fig. 12) directly behind the dogleg/collimator. The ORS is followed by sFLASH [43], the experimental setup for seeding the FEL with the radiation produced by the generation of higher harmonics of Ti:Sa laser in rare gases. The laser beam together with high harmonics is sent from the laser laboratory to the tunnel, where it is injected into the electron beam line through the dogleg. In the case of successful lasing of sFLASH, the amplified VUV radiation is supposed to be outcoupled by a mirror and transported to the experimental hutch (at the same time laser beam for pump-probe experiments is transported there from the laser laboratory). The distance between the dogleg and the mirror is about $30 \mathrm{~m}$.

We note that this infrastructure can be used for production of attosecond pulses in the LSCA and using them for experiments in the hutch. The total length of LSCA is

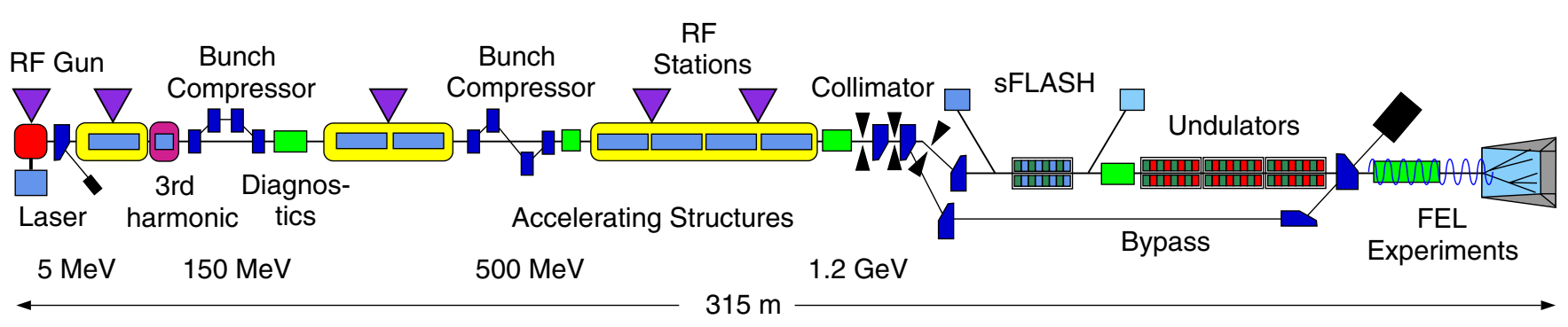

FIG. 12. FLASH layout [40]. The laser lab and the experimental hutch are shown as blue squares.
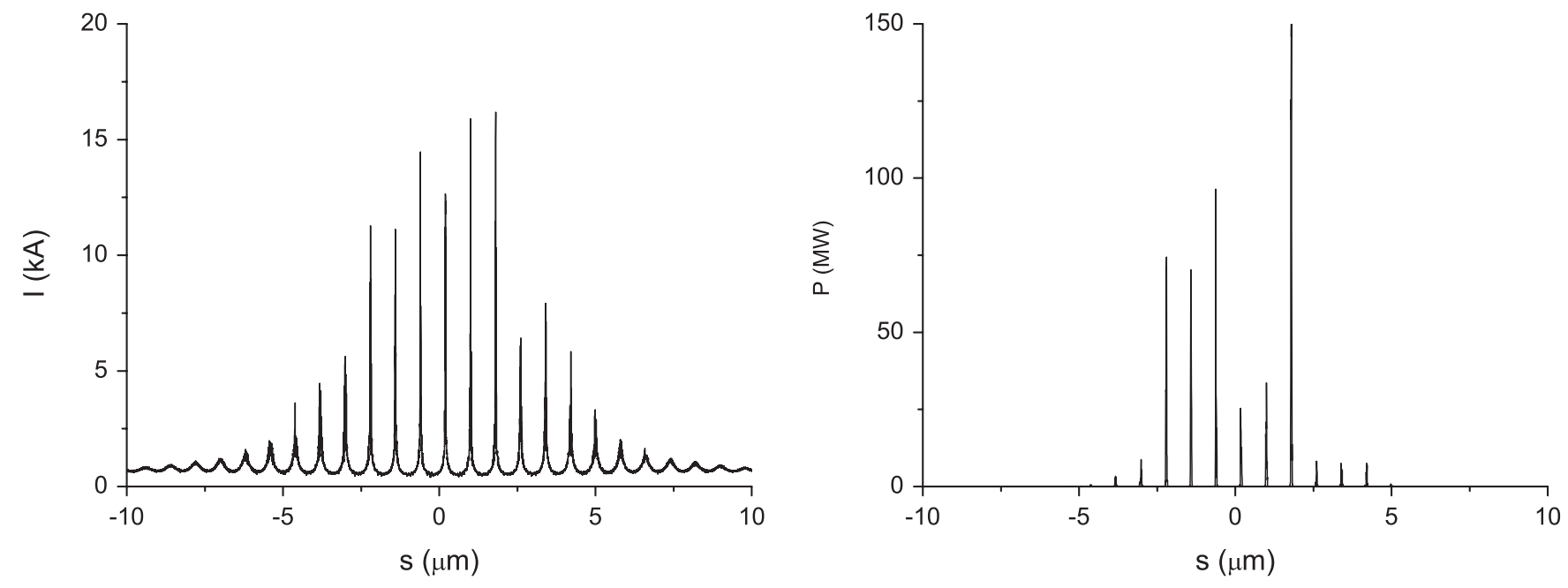

FIG. 13. Modulated electron bunch at the exit of the fourth chicane (left) and power of the radiation from the undulator (right) for the case of a relatively long pulse (35 fs FWHM) from the Ti:Sa laser. The calculations were performed with LOSCA and FAST. 
supposed to be about $14 \mathrm{~m}$, so that it can substitute one of the two existing setups. Experiments can be started with the existing Ti:Sa laser (that delivers 35 fs long pulses with a pulse energy of up to $50 \mathrm{~mJ}$ [43]) for modulation of the electron beam, in this case one will produce a train of attosecond soft x-ray pulses. We have simulated this situation with LOSCA and FAST, the results are presented in Fig. 13. To get required amplitude of energy modulations, we used only $15 \mathrm{~mJ}$ out of available laser pulse energy.

Later the laser can be upgraded in order to produce $5 \mathrm{fs}$ long pulses with pulse energy about $3 \mathrm{~mJ}$ which would allow one to obtain an isolated attosecond x-ray pulse. The attosecond pulses with the help of the outcoupling mirror can be transported to the existing experimental hutch where they can be used together with synchronized few femtosecond long powerful laser pulses.

As a possible option, one can consider seeding FLASH undulator with an attosecond pulse. In this case the outcoupling mirror is moved out, and the chicane at that position is used to slightly delay the electron bunch so that the radiation pulse is parked on the fresh part of the bunch. Despite the transverse size of the radiation at the entrance of FLASH undulator is much larger than that of the electron beam, an effective seed power is still much larger than an effective power of shot noise. During amplification the radiation pulse would be stretched, so that finally pulse duration would be comparable to FEL coherence time (about $1 \mathrm{fs}$ ).

Finally we note that the attosecond scheme, described in this paper, can also be used at FLASH II [44], FERMI@Elettra [45], and similar facilities, as well as at soft X-ray beam lines of large x-ray FEL facilities like SASE3 beam line of the European XFEL [46].

[1] E. L. Saldin, E. A. Schneidmiller, and M. V. Yurkov, Nucl. Instrum. Methods Phys. Res., Sect. A 483, 516 (2002).

[2] E. L. Saldin, E. A. Schneidmiller, and M. V. Yurkov, Nucl. Instrum. Methods Phys. Res., Sect. A 528, 355 (2004).

[3] Z. Huang et al., Phys. Rev. ST Accel. Beams 7, 074401 (2004).

[4] M. Venturini, Phys. Rev. ST Accel. Beams 11, 034401 (2008).

[5] D. F. Ratner, A. Chao, and Z. Huang, in Proceedings of the FEL2008 Conference, Gyeongju, Korea, p. 338 [http:// www.jacow.org].

[6] H. Loos et al., Proceedings of FEL2008 Conference, Gyeongju, Korea, p. 485 [http://www.jacow.org].

[7] S. Wesch et al., Proceedings of FEL2009 Conference, Liverpool, UK, p. 619 [http://www.jacow.org].

[8] A. Lumpkin et al., Phys. Rev. ST Accel. Beams 12, 080702 (2009).

[9] M. Clemens et al., DESY Report No. TESLA-FEL-200902, Hamburg, 2009.
[10] Z. Huang et al., Phys. Rev. ST Accel. Beams 13, 020703 (2010).

[11] E. A. Schneidmiller and M. V. Yurkov, Phys. Rev. ST Accel. Beams 13, 110701 (2010).

[12] E. L. Saldin, E. A. Schneidmiller, and M. V. Yurkov, Opt. Commun. 212, 377 (2002).

[13] E. L. Saldin, E. A. Schneidmiller, and M. V. Yurkov, Opt. Commun. 237, 153 (2004).

[14] A. A. Zholents and W. M. Fawley, Phys. Rev. Lett. 92, 224801 (2004).

[15] P. Emma, Z. Huang, and M. Borland, in Proceedings of the FEL2004 Conference (Comitato Conferenze Elettra, Trieste, Italy, 2004), p. 333 [http://www.jacow.org].

[16] A. A. Zholents and G. Penn, Phys. Rev. ST Accel. Beams 8, 050704 (2005).

[17] E. L. Saldin, E. A. Schneidmiller, and M. V. Yurkov, Phys. Rev. ST Accel. Beams 9, 050702 (2006).

[18] Y. Ding et al., Phys. Rev. ST Accel. Beams 12, 060703 (2009).

[19] D. Xiang, Z. Huang, and G. Stupakov, Phys. Rev. ST Accel. Beams 12, 060701 (2009).

[20] W. Ackermann et al., Nat. Photon. 1, 336 (2007).

[21] K. Tiedtke et al., New J. Phys. 11, 023029 (2009).

[22] By a "drift space" or a "drift" we mean in this paper a focusing channel, in which LSC effect accumulates. It is a drift from a point of view of longitudinal dynamics, but not a transverse one.

[23] Note that the laser beam can be incoupled either through the previous chicane where the electron beam has a transverse offset, or through some bending system upstream of the first cascade of LSCA.

[24] I. Zagorodnov and M. Dohlus, Phys. Rev. ST Accel. Beams 14, 014403 (2011).

[25] I. Zagorodnov, in Proceedings of the FEL2010 Conference, Malmö, Sweden [http://www.jacow.org].

[26] K. Flöttmann, ASTRA homepage, http://www.desy.de/ mpyflo, 2000.

[27] G. Pöplau and K. Flöttmann, in Proceedings of ICAP2006, Chamonix, France, p. 136 [http://www.jacow.org].

[28] E. L. Saldin, E. A. Schneidmiller, and M. V. Yurkov, The Physics of Free Electron Lasers (Springer, Berlin, 2000).

[29] G. Gelolni et al., Nucl. Instrum. Methods Phys. Res., Sect. A 578, 34 (2007).

[30] M. Dohlus and T. Limberg, in Proceedings of the FEL2004 Conference (Ref. [15]), p. 18 [http://www .jacow.org].

[31] M. Dohlus and T. Limberg, CSRTRACK Version 1.2 User's Manual, DESY, 2007.

[32] E. L. Saldin, E. A. Schneidmiller, and M. V. Yurkov, Nucl. Instrum. Methods Phys. Res., Sect. A 417, 158 (1998).

[33] M. Dohlus, DESY Report No. TESLA-FEL-2003-05, Hamburg, 2003.

[34] E. L. Saldin, E. A. Schneidmiller, and M. V. Yurkov, Nucl. Instrum. Methods Phys. Res., Sect. A 490, 1 (2002).

[35] S. Heifets, G. Stupakov, and S. Krinsky, Phys. Rev. ST Accel. Beams 5, 064401 (2002).

[36] Z. Huang and K.-J. Kim, Phys. Rev. ST Accel. Beams 5, 074401 (2002). 
[37] It is defined as $\left\langle\left|\int_{-\infty}^{\infty} I(t) e^{i \omega t} d t\right|^{2}\right\rangle^{1 / 2} / \int_{-\infty}^{\infty} I(t) d t$.

[38] E. L. Saldin, E. A. Schneidmiller, and M. V. Yurkov, Nucl. Instrum. Methods Phys. Res., Sect. A 429, 233 (1999).

[39] A. M. Kondratenko and E. L. Saldin, Part. Accel. 10, 207 (1980).

[40] http://flash.desy.de.

[41] E. L. Saldin, E. A. Schneidmiller, and M. V. Yurkov, Nucl. Instrum. Methods Phys. Res., Sect. A 539, 499 (2005).

[42] G. Angelova et al., Phys. Rev. ST Accel. Beams 11, 070702 (2008).
[43] J. Bödewadt et al., in Proceedings of the FEL2010 Conference, Malmö, Sweden (Ref. [25]) [http://www .jacow.org].

[44] B. Faatz et al., in Proceedings of the IPAC'10 Conference, Kyoto, Japan (ICR, Kyoto, 2010), p. 2152 [http://www .jacow.org].

[45] S. Di Mitri et al., Nucl. Instrum. Methods Phys. Res., Sect. A 608, 19 (2009).

[46] M. Altarelli et al., Technical Design Report No. DESY 2006-097, Hamburg, 2006 [see also http://xfel.desy.de]. 